\title{
Parkinson disease and use of stem cells for therapeutic approaches of Parkinson disease
}

\author{
Fehmida Farid Khan ${ }^{1}$, Tania Tanveer ${ }^{1}$, Asma Gul ${ }^{1}$ \\ ${ }^{1}$ (Department of Bioinformatics \& Biotechnology, International Islamic university Islamabad, H-10 Campus \\ Islamabad Pakistan).
}

\begin{abstract}
Parkinson's disease involves a progressive loss of midbrain dopaminergic neurons in the substantia nigra, which causes motor symptoms such as dysfunctional bradykinesia, rigidity and tremor. Genetic mutations in Parkinson disease are infrequent and important because they represent potential therapeutic targets. Unfortunately, there is currently no treatment to stop the progression of the disease but only few improvements in symptoms are accessible by current treatments based on levodopa and dopaminergic therapy. Embryonic stem cells, Neural stem cells, Mesenchymal stem cell and induce pluripotent stem cell are a good source for dopaminergic neurons and can be used for cellular therapeutic approaches. Mesenchymal stem cells are thought to be among the easiest to harvest stem cells from individual sources. Embryonic stem cells and induced pluripotent stem cells have more advantages than other two. Embryonic stem cells remain highly proliferative after in vivo expansion. They can generate dopaminergic neurons and survive after transplantation. Along with it, induced pluripotent stem cells generate unlimited Parkinson's disease patient specific cells. They show some degree of functional recovery after transplantation. Hence, these two have potential to enhance the success rate of clinical trial in Parkinson disease and to tolerate adverse effects of transplantation. This review will provide an insight into therapeutic approaches of Parkinson disease by the help of different types of stem cells.
\end{abstract}

Keywords: Bone marrow mesenchymal stem cells, Human embryonic stem cell Induced pluripotent stem cell, Myocyte-specific enhancer factor 2C, Neural stem cells..

\section{Introduction}

Parkinson's disease (PD) is the second most common neurodegenerative disorder affecting $1-2 \%$ of the population aged over 65 years and reaching a prevalence of nearly $4 \%$ in people over 85 years. The main clinical symptoms of the disease are resting tremor, rigidity, bradykinesia, and postural instability $[1,2]$. The pathological features of the disease are loss of dopaminergic cells in the substantia nigra pars compacta and the presence of Lewy bodies (LB) and Lewy neuritis (collectively known as Lewy-related pathology) among vulnerable populations of neurons [3]. Symptoms appear when $50-70 \%$ of nigrostriatal dopaminergic neurons have been lost. Thus, the population of undiagnosed asymptomatic patients is probably considerable [4]. Unfortunately, there is currently no treatment to stop the progression of the disease but only few improvements in symptoms are accessible by current treatments based on levodopa and dopaminergic therapy [1]. In most cases, PD is multifactorial, probably resulting from a combination of polygenic inheritance, environmental exposures and gene-environment interactions. Approximately $20 \%$ of PD patients report a family history of the disease and monogenic forms of PD are relatively rare [5]. Genetic mutations in PD are sporadic and only about $10 \%$ of all cases of Parkinson's disease [6]. There are at least eighteen loci [7], and eleven genes associated with PD as shown in Table 1 [8]. Mutations in the genes of these six loci (SNCA, LRRK2, DJ1, PINK1, ATP13A2 and PRKN) have been definitively shown to cause familial parkinsonism [19]. In addition, common polymorphisms within 2 of these same genes (SNCA and LRRK2) and variation in 2 other genes not assigned to a PARK locus, (GBA and MAPT) are now well-validated risk factors for PD [20].

\section{Autosomal Dominant PD}

\subsection{PARK4 and PARK1: $\alpha$-Synuclein $(S N C A)$}

SNCA is located on chromosome 4q22.1, has 6 exons, and encodes a 140 -amino acid protein [21]. It is expressed in the mammalian brain and enriched in presynaptic nerve terminals [3]. The role of the SNCA gene is important in PD because the alpha-synuclein protein is the main component of Lewy bodies [19]. Mutations in the $\alpha$-Syn gene are rare include entire locus multiplications and point mutations [22]. Familial autosomal dominant forms of PD have been documented in families with SNCA mutations or gene duplication/ triplications as well as in families with mutations in proteins regulating the ubiquitin-proteasome pathway [9]. The Ala53Thr mutation has been found in a several families of Greek descent previously, Ala30Pro and Glu46Lys have been demonstrated in single families of German and Spanish origin, respectively [23, 24]. Patients with SNCA duplications often have a classical PD phenotype, while cases involving triplications 
display more severe phenotypes, according to a direct correlation between the dose of SNCA gene and disease severity [23].

Table I: Loci and genes associated with PD.

\begin{tabular}{|c|c|c|c|}
\hline Locus & Gene & Inheritance & References \\
\hline PARK1/PARK4 & SNCA & AD & {$[10]$} \\
\hline PARK8 & LRRK2 & AD & {$[11]$} \\
\hline PARK5 & UCH-L1 & AD & {$[12]$} \\
\hline PARK11 & GIGYF2 & AD & {$[13]$} \\
\hline PARK13 & OMI/ HTRA2 AR & AR & {$[14]$} \\
\hline PARK2 & PARKIN & AR & {$[16]$} \\
\hline PARK6 & PINK1 & AR & {$[17]$} \\
\hline PARK7 & DJ-1 & AR & {$[18]$} \\
\hline PARK9 & ATP13A2 & AR & {$[18]$} \\
\hline PARK14 & PLA2G6 & FBXO7 & \\
\hline PARK15 & &
\end{tabular}

*AD (Autosomal dominant) AR (Autosomal recessive)

\subsection{PARK8: LRRK2}

LRRK2 is a large gene that consists of 51 exons. It is located on chromosome $12 \mathrm{p} 12$ and encompasses $144 \mathrm{~kb}$ [25], encodes the 2527-amino acid cytoplasmic protein leucine-rich repeat kinase 2 (LRRK2) that consists of a leucine-rich repeat [26]. Alterations in Leucine-rich repeat kinase 2 (LRRK2) are commonly associated with Parkinson's disease, most of these patients do not exhibit a phenotype of Lewy and it is thought that aggregation protein may be a disease modifier [10]. Mutations in the LRRK2 gene are the most common known cause of late-onset autosomal dominant with a mutation frequency ranging from $2 \%$ to $40 \%$ in different populations [27]. There are more than 50 different missense and nonsense mutations reported in LRRK2 [26]. These six mutations (R1441C, R1441G, R1441H, Y1699C, G2019S, and I2020T) are disease-causing mutations. The most frequent and best-studied mutation is c.6055G. A (p.G2019S) that accounts for as many as $40 \%$ of cases of Arab origin [27], 1\%-7\% of PD patients of European origin [28, 29], and about 20\% of Ashkenazi Jewish patients [30].

\subsection{PARK2: Parkin}

\section{Autosomal Recessive PD}

Parkin is a 65-amino acid protein that belongs to the "ring between ring fingers" (RBR) family of E3 ubiquitin ligases [31]. It is located on chromosome 6q26 and contains 12 exons and encompasses approximately $1.38 \mathrm{Mb}[14,32]$. PARK2 Parkinsonism is autosomal recessive and usually of early onset [33]. Pathologically, symptoms are severe neuronal loss in the substantia nigra and gliosis, whereas the locus coeruleus is much less severely involved and usually no LBs are seen [34, 35]. Reported mutations in PARK2 are more than 100 including missense and nonsense mutations, as well as rearrangements, exonic deletions and duplications [36, 37]. The most frequent mutations are deletions of exon 4 (n $1 / 428)$, deletions of exon 3 (n $1 / 427$ ), deletions of exons 3 to 4 (n $1 / 423)$, a point mutation in exon7 (924C>T; $1 \frac{1}{4} 38$ ) and a single base pair deletion in exon 2(255/256delA; $n$ 1/4 17) [38].

\subsection{PARK7: DJ1}

DJ-1 is a mitochondrial protein consisting of 189 amino acids [39], involved in the protection against oxidative stress and forms a complex with PINK1 and parkin to prop up ubiquitination and degradation of parkin substrates, including parkin itself [40]. It is located on chromosome 1p36.23 and has a transcript length of 949 bps with 7 exons [41]. In DJ1-related Parkinsonism, mutations are found in the homozygous or compound heterozygous state, putatively resulting in a loss of protein function [42]. The structural perturbations of DJ1 protein may be caused by mutation of L166P, E64D, M26I, A104T, and D149A that lead to global destabilization, heterodimer formation, unfolding of the protein structure, or reduced antioxidant activity [43].

\subsection{PARK6: PINK1}

PINK1 is a kinase with an N-terminal mitochondrial targeting sequence and has a serine/threonine protein kinase domain [44], provides protection against mitochondrial dysfunction, and regulates mitochondrial morphology via fission/fusion machinery [45]. It is located on chromosome 1p36.12, has 8 exons, and encodes a protein with 581 amino acids [44]. PARK6 was first mapped in a large consanguineous Italian family with 
autosomal recessive, early onset PD [46]. The first mutations discovered were the G309D missense and a W437X truncating mutation found in the families of Italian and Spanish origin [44, 15].The majority of reported PINK1 mutations are either nonsense or missense mutation [47]. Most of the reported PINK1 point mutations are in a highly conserved amino acid position in the protein kinase domain [48]. The pathogenic mutation (A217D) is located in the highly conserved adenosine triphosphate orientation site of the PINK1 kinase domain [49].

\subsection{PARK9: ATP13A2}

ATP13A2 encodes a large lysosomal P-type ATPase with 1180 amino-acids [50], likely involved in the regulation of intracellular manganese homeostasis [51].It is mainly expressed in brain tissue with the highest levels in ventral midbrain (which includes the substantia nigra). It is located on chromosome 1p36 and has 29 exons [50]. Homozygous and compound heterozygous mutations in the ATP13A2 have been demonstrated in a Jordanian family [52]. A homozygous missense mutation (G504R) has been identified in one sporadic case from Brazil with juvenile parkinsonism. A single novel missense mutation (T12M or G533R) in two Italian patients with early onset PD without atypical features, raising the question of whether heterozygous ATP13A2 mutation carriers are at increased risk of PD [53].

\section{Genetic Risk Factors for PD}

GIGYF2, also called TNRC15 (Trinucleotide Repeat Containing 15) corresponds to the PARK11 locus, previously identified by a whole genome linkage analysis in a population of familial PD [54]. GIGYF2 contains the GYF motif that binds to a proline-rich Grb10 adaptor protein [55], and potentially regulates cellular responses to insulin and insulin-like growth factor. Mostly in sporadic PD cases no disease-causing mutations were found in other European populations [56]. Omi/Htra2, a mitochondrial-targeted serine protease released into the cytosol during apoptosis. Biological and genetic evidence showed that it has been implicated in PD pathogenesis. Subsequently, a G399S mutation and an A141S risk factor were identified in a German casecontrol study [13], but no relations between A141S or G399S and PD were found in other studies [57]. A homozygous R378G variation in FBXO7 on chromosome 22 was found in an Iranian pedigree with a rare autosomal recessive parkinsonian-pyramidal syndrome. FBXO7 is a member of the F-box family of proteins active in the ubiquitin-proteasome protein degradation pathway [58, 59]. Homozygous missense mutations were found in a phospholipase A2 gene (PLA2G6) on chromosome 22 in two unrelated Pakistani families with recessive adult-onset levodopa-responsive dystonia-parkinsonism, encoding a calcium-independent group VI phospholipase A2 [60]. Heterozygous mutations in the glucocerebrosidase (GBA) gene (encodes the enzyme glucocerebrosidase), however, have been validated as genetic susceptibility factors. Gaucher disease (GD), an autosomal recessive glycolipid storage disorder with multisystemic manifestations, including involvement of the liver, spleen, bone marrow, lungs, and nervous system, is caused by loss of function mutations in the GBA gene [61]. The causal genes at several loci have not yet been identified (PARK12, chromosome Xq) [62], or the role of the candidate genes at these loci is still controversial (PARK3 and PARK10) [63, 64]. Recent Genome-wide association studies have nominated 3 new susceptibility loci (PARK16, 17, 18) [25].

\section{Use of stem cells in Parkinson disease}

Pharmacological agents do not restore DA neurons in PD patients but used to treat symptoms [65]. Stem cell-based methods could be used therapeutically to restore function in neurodegenerative disease [66].Such cell-based therapies require protocols for the differentiation of stem cells into neural progenitor cells and more directly towards a specialized regional identity of neurotransmitters, such as dopaminergic midbrain neurons [67]. Different types of stem cells can be used in PD as shown in Table II.

Table II: Different sources and types of stem cells are used in PD patients.

\begin{tabular}{|c|c|}
\hline Source & Reference \\
\hline Mesenchymal stem cell (MSCs) & {$[68]$} \\
\hline Neural stem cells (NSCs) & {$[69]$} \\
\hline embryonic stem cell (ESCs) & {$[70]$} \\
\hline Induced pluripotent stem cells (iPSCs) & {$[71]$} \\
\hline
\end{tabular}

\subsection{Mesenchymal stem cell}

MSCs are adult stem cells that belong to the mesodermal lineage and are usually found in the bone marrow as bone marrow mesenchymal stem cells (BMSCs) [72]. It can also be isolated from other mesenchymal tissues, such as umbilical cord, dermis, adipose tissue, and peripheral blood [73]. The great advantage of BMSCs is that they are easily available through aspiration of the patient's bone marrow, so that the use of BMSCs evades ethical issues, facilitating their application both for auto- and allo-transplantation. BMSCs are 
also easily expanded on a large scale, which is very convenient for clinical use (e.g., 20 to $100 \mathrm{~mL}$ of bone marrow aspirate provides 107 BMSCs within several weeks) [74]. MSCs can be obtained from patients for autocell transplantations well as from healthy donors for allo-transplantation by using mesenchymal tissues such as fat, bone marrow, and umbilicalcord (Fig.1). Therefore, MSCs are a realistic source of cell for regenerative medicine [75].

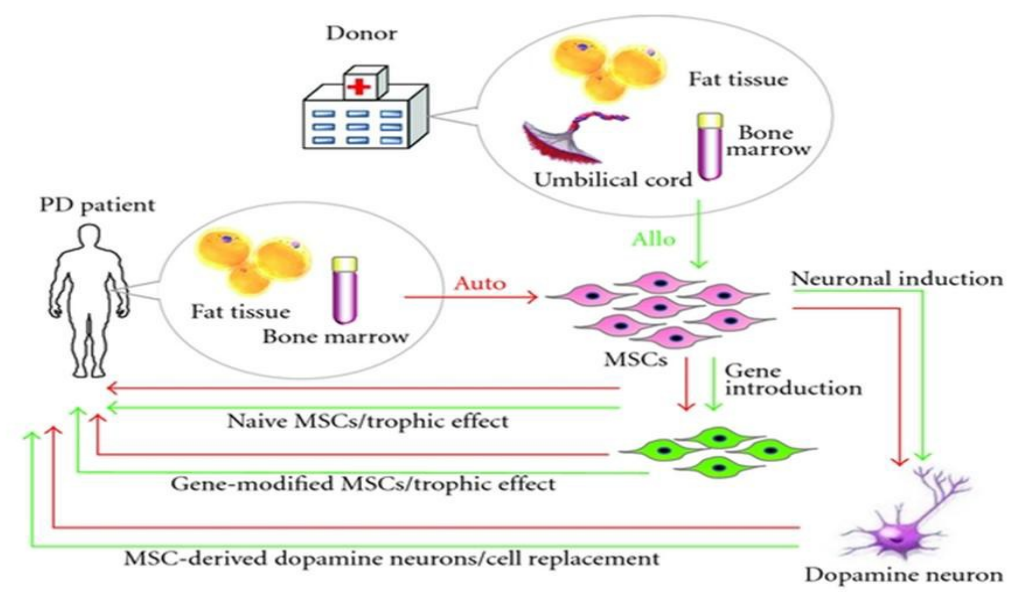

Figure.1. Strategy for MSC transplantation in PD patients. MSCs can be obtained from fat tissue or bone marrow aspirates of Parkinson's disease (PD) patients and are applicable for autocell transplantation, whereas healthy donors for allo cell transplantation. Naive MSCs can be directly transplanted into the striatum of PD patients, but this treatment exerts temporary trophic effects. Gene-introduced MSCs also have trophic effects for the replenishment of lost cells. MSCs are able to be induced into dopamine neurons that will contribute to the functional recovery of PD [75].

BMSCs have trophic effects that are mediated by the various types of trophic factors and cytokines they produce. Therefore, naive adult BMSCs implanted in the striatum induce partial but not strong recovery of the dopamine pathway [76]. Mesenchymal stem cells derived from bone marrow have been proposed as potential source for transplantation in PD. When mice model was used to test the effect of MSCs, it was observed that non-differentiated murine MSCs are able to differentiate into tyrosine hydroxylase-positive neurons and they showed improved motor performance. It has been proved that human and rat MSCs can be a source for the production of cells with dopaminergic properties and motor function can be improved in animal model by the transplantation of these cells. In a recent study, no hostile effect has been found during a clinical trial in PD patients in which unilateral transplantation of autologous bone marrow-derived MSCs was done into the sublateral ventricular zone [77]. Adipose tissue, which is easily achieved by liposuction, also contains large quantities of MSCs called adipose stem cells (ADSCs).Because of the ability of ADSCs and umbilical cord mesenchymal stem cells (UC-MSCs) to differentiate into other cell types and to proliferate; these cells are considered to be a practical source for cell-based therapies [78].

In a study human umbilical MSCs showed the potential for the treatment of Parkinson's disease. The success rate was found $12.7 \%$ when human MSCs isolated from Wharton's jelly of the umbilical cord were induced to transform into dopaminergic neurons in vitro through step wise culturing in neuron-conditioned medium. This success rate was characterized by positive staining for tyrosine hydroxylase $(\mathrm{TH})$, the rate limiting catecholaminergic synthesizing enzyme, and dopamine being released into the culture medium. When such cells were transplanted into the striatum of rats previously made Parkinsonian by unilateral striatal lesioning with dopaminergic neurotoxin 6-hydroxydopamine, the transplantation partially corrected the lesion induced amphetamine-evoked rotation. Those transplanted cells showed viability for at least 4 months [79].Protective effect on progressive dopaminergic neural loss was observed in vitro and in vivo by human MSCs. This treatment decreased MG-132 induced dopaminergic neuronal loss with a significant reduction of caspase-3 activity. Afterwards, when a rat model was tested, hMSCs treatment in MG-132 treated rats dramatically reduced the decline in the number of tyrosine hydroxylase immune reactive cells, showed almost $50 \%$ upsurge in the survival of TH immunoreactive cells in the substantianigra. Also, hMSCs treatment significantly decreased OX-6 immunoreactivity and caspase-3 activity [80].

\subsection{Embryonic stem cell}

Embryonic stem (ES) cells have attracted great attention as an alternative source for the generation of dopamine neurons because they can be constantly expanded with high potential for differentiation. They are pluripotent stem cells, following induced differentiation they are able to form all three embryonic germ layer 
[81]. Dopaminergic neurons can be produced from various stem cell types as shown in Fig.2. ES cells can produce a sufficient number of dopamine neurons for transplantation therapy. Their clinical application is limited because of their ability to form tumors. Even if ES cells seem fully differentiated into dopamine neurons in vitro before transplantation, they still associated with the risk of tumorigenesis [82]. Myocyte enhancer factor 2 (MEF2C) directs the differentiation of mouse ESC-derived neural precursors into neurons.MEF2C confines $\mathrm{hESCs}$ to the neuronal lineage and that this attribute can be used to generate neurons and avoid tumor formation when used for cell-based therapies [83]. ESCs can give rise to any type of cell in the body including dopaminergic neurons due to their pluripotency and highly proliferative property. Human and rodent ESCderived dopaminergic neurons have been shown to survive transplantation into the striatum of PD rats and generate some degree of functional recovery. Nevertheless, studies have shown that the survival of ESC-derived dopaminergic neurons post transplantation is relatively low [76]. In a study it was demonstrated that hESCs differentiated into dopaminergic neurons when co-cultured with PA6 cells. Almost $87 \%$ of hES colonies contained tyrosine hydroxylase (TH) positive cells, and a high percentage of the cells in most of the colonies expressed TH after three weeks of differentiation [84].

Rodriguez-Gomez showed the survival of neurons derived from mouse ES cells for over 32 weeks when transplanted into an animal model. Moreover, maintained midbrain markers and sustained behavioral effects were also observed for 32 weeks. When microdialysis was performed in grafted animals it was observed that dopamine (DA) release was induced by depolarization and pharmacological stimulants. Positron emission tomography measured the expression of presynaptic dopamine transporters in the graft and also showed that the number of postsynaptic DA D2 receptors was normalized in the host striatum. Therefore the data suggested that ES-cell derived neurons showed Dopamine release and reuptake and stimulate appropriate postsynaptic responses for long periods after implantation [85]. The hESC-based therapies face several obstacles, including death of engrafted cells, failure of incorporation into host brain, lack of differentiation into appropriate neuronal cell types, and formation of tumors after transplantation [86].

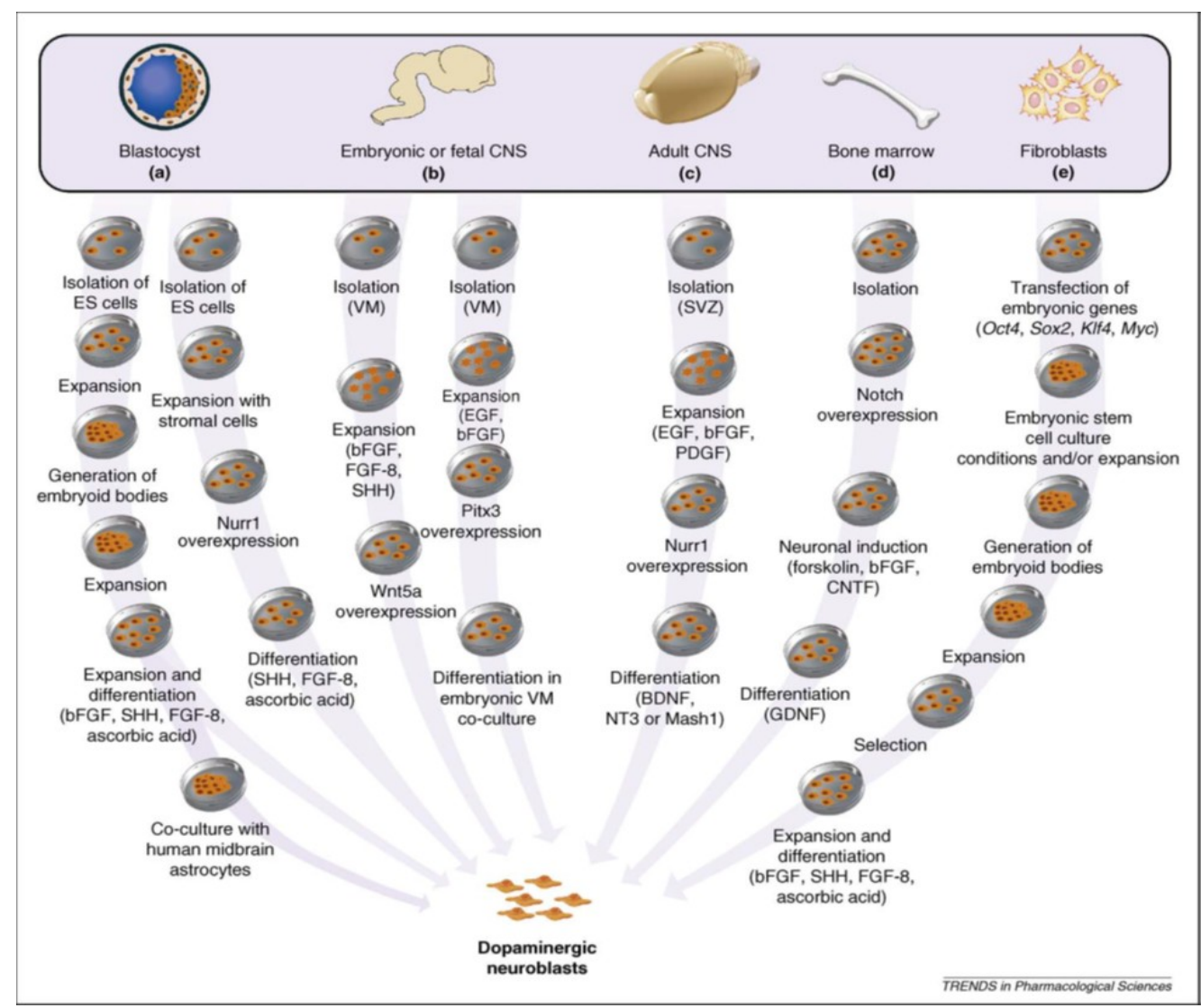

Figure.2. Production of neuroblasts from stem cells for transplantation in PD using different protocols. (a) growth of embryonic stem cells as embryoid bodies and differentiation with several factors. (b) progenitors of embryonic VM and expansion with trophic factors. (c) subventricular-zone derived cells from adult brain after expansion as neoruspheres with growth factors. (d) bone marrow stem cells after Notch overexpansion. (e) Fibroblast-derived iPS cells after culturing as embryonic stem cells [82]. 


\subsection{Neural stem cells}

Neural stem cells (NSCs) are an attractive source for cell replacement therapy for PD because they have the ability to differentiate into dopamine neurons [87]. Neural stem cells are a source of autologous dopamine neurons and can be used for patient and disease-specific pathogenic studies [69]. They can be harvested both from fetal and adult central nervous system tissues. Adult NSCs have lower capacity for differentiation than fetal NSCs [88]. Chromatin-modifying agents can increase differentiation of adult Sub ventricular zone (SVZ) NSCs without altering their capacity to differentiate into region-specific neuronal phenotypes. NSCs epigenetic modifications indicate an important role in regulating the differentiation of adult NSC [89].Chromatin-modifying agents TSA (trichostatin A) and TSA in combination with (5Aza-2deoxycytidine), reactivate specific pluripotency-associated genes such as Oct4 and Klf-4 in adult SVZ NSCs. These agents induced partial demethylation of Oct4 promoter, thus promoting a dedifferentiation toward a nascent neural stage. Oct4 overexpression in association with the chromatin-modifying agent valproic acid (VPA) induced adult SVZ NSC reprogramming into iPSCs. Such Oct4-reprogrammed NSCs can then successfully differentiate into midbrain DA neurons. NSC self-renewal and long-term proliferation can be increased by Oct-4 overexpression without reprogramming to an ESC state in the presence of epidermal growth factor (EGF) and FGF-2 [90].

Fetal brain neural SC derived dopaminergic neurons are associated with lower risk of tumor formation and immune rejection than ESCs. In a recent study, it has been showed that, non-differentiated NSCs implanted into PD primates survived, migrated and has a functional impact. A small number of NSC progeny differentiated into Dopamine phenotypes. Dopaminergic yield in fetal NSC differentiation in vitro has been enhanced by the use of development signals such as sonic hedgehog, Wnt5a [76]. Therapeutic potential of neural stem cells in transplantation treatment against PD the midbrain-derived neural stem cells (mNSCs) were studied by in vitro culture. Their proliferative, differentiation and neurotrphic properties in comparison with hippocampus-derived NSCs (hNSCs) were studied. It was observed that mNSCs have lower proliferative rate than that of hNSCs but had higher cell survival capacity in serum free culture. Secondly, in differentiation culture, mNSCs demonstrated similar Tuj-1+immatureneuronal differentiation but higher Nurr1+ and tyrosine hydroxylase $(\mathrm{TH})+$ cell fate in comparison with hNSCs. Moreover, mNSCs expressed various neurotrophic factors like brain derived neurotrophic factor (BDNF), glial-derived neurotrophic factor (GDNF), cerebral dopamine neurotrophic factor (CDNF) and DJ-1, that actively function in dopaminergic neuronal maintainence or neuroprotection with their slightly same or different levels comparatively with hNSCs. Conclusively, mNSCs have unique cell proliferation, cell survival, dopaminergic neuronal differentiation and neurotrophic properties, which suggests that midbrain-derived NSCs may be an ideal cell source for therapeutic cell transplantation in human beings [91].

\subsection{Induced pluripotent stem}

Another favourable source of SCs is adult fibroblasts that are reprogrammed to so called induced plutipotent SCs (iPSCs) and then differentiated to dopaminergic neurons. With this iPSC technology the possibility of generating an unlimited source of PD patient specific dopaminergic neurons has been raised [76]. The iPS cells, whose properties are similar to those of ES cells, can be generated from adult human cells, such as dermal fibroblasts, by introducing genes such as Sox2, Oct3/4, Klf-4, and c-Myc. The iPS cells can generate cells of all three germ layers and have unlimited proliferative activity, but their clinical application is limited by their tumorigenicity. The iPS cells can be induced to form dopamine neurons, but the efficiency of induction is generally lower than that of ES cells and the quality of the cells is not homogenous [92].The majority of human IPSC lines can be generated using lentiviral and retroviral methods, which are known to generate multiple chromosomal integrations and possible genetic dysfunction [93]. A major concern about the clinical application of hiPSCs is the potential residual expression and reactivation of exogenous reprogramming genes, some of which may be asscociated in oncogenesis pathways [94].Terminal differentiation of hiPSCs into some cell lineages may also alter or block by residual reprogramming gene expression. The hiPSCs generated by the lentiviral method exhibit neural differentiation capacity with significantly reduced efficiency and increased variability compared with hESCs [71]. High levels of a high activity form of human Sonic hedgehog (SHH) and exposure to FGF8a and WNT1 may be sufficient to direct the fate of human ES/iPS cells towards VM DA neurons [95]. The iPS cells do not cause the same immune rejection risks when used therapeutically, since they are autologous cells that are unique to each patient [80].

Dopmaninergic neurons were first generated from mouse iPSCs transplanted into the striatum of a rat PD model and shown to improve functional deficits. In recent studies, dopaminergic neurons have been produced from iPSCs derived from fibroblasts in adult humans and PD patients. Those neurons survived transplantation into the striatum of PD rodents and produced some degree of functional recovery. Major gains with the use of iPSCs are that PD patient-specific dopaminergic neuroblasts could minimize the immune reactions and eliminate the ethical issues associated with the use of human ESCs. Still, as with ESCs, the risk of 
tumor formation needs to be minimized before iPSC-derived dopaminergic neurons can be considered as an option for transplantation in a clinical setting in PD [76].

\section{Conclusion}

Parkinson's disease is a degenerative disorder of the central nervous system in which the death of dopamine-generating cells in the substantia nigra, occur. Mutations occur in different genes and these mutations are important because they represent potential therapeutic targets. Different types of stem cells including embryonic stem cell, neural stem cells, Mesenchymal stem cell and iPSC can be used for cellular therapeutic approaches. Multiple factors can differentiate these cells into dopamine neurons which are used for damage neurons of PD patients. Factors which are used for differentiation depend upon the type of stem cell. There are chances of tumor formation after transferring dopamine neurons into Parkinson Disease patient. Embryonic stem cells and induced pluripotent stem cells have more advantages than other two. Embryonic stem cells remain highly proliferative after in vivo expansion. They can generate dopaminergic neurons and survive after transplantation. Along with it, induced pluripotent stem cells are the good source for generation of unlimited Parkinson's disease patient specific cells and show some degree of functional recovery after transplantation. They also minimize immune reactions. Major research efforts will be needed for the development of a clinically competitive SC-based therapy, which for the first time opens up the possibility for an effective restorative treatment for PD patients.

\section{References:}

[1] B. Thomas and MF. Beal, Molecular insights into Parkinson's disease. F1000 Medicine Reports, 3, $2011,7$.

[2] MC. Rijk, LJ. Launer ,K. Berger, MM. Breteler, JF. Dartigues, M. Baldereschi, L. Fratiglioni, A. Lobo, J. Martinez-Lage, C. Trenkwalder and A. Hofman, Prevalence of Parkinson's disease in Europe: a collaborative study of population-based cohorts. Neurologic Diseases in the Elderly Research Group, Neurology, 54(11 suppl 5), 2000, S21-S23.

[3] JM. George, The synucleins. Genome Biology, 3(1), 2002, REVIEWS 3002.

[4] AH. Schapira, Present and future drug treatment for Parkinson's disease, Journal of Neurology, Neurosurgery. And Psychiatry, 76(11), 2005, 1472-1478.

[5] MJ. Farrer, Genetics of Parkinson disease: paradigm shifts and future prospects. Nature Reviews Genetics, 7(4), 2006, 306-318.

[6] W. Dauer and S. Przedborski, Parkinson's disease: mechanisms and model. Neuron, 39(6), 2003, 889-909.

[7] TH. Hamza, CP. Zabetian, A. Tenesa, A. Laederach, J. Montimurro, D. Yearout, DM. Kay,KF. Doheny,J. Paschall,E. Pugh,VI. Kusel, R. Collura, J. Roberts, Common genetic variation in the HLA region is associated with late-onset sporadic Parkinson's disease. Nature Genetics, 42(9), 2010, 781-785.

[8] F. Coppede, Genetics and Epigenetics of Parkinson's disease. The scientific World Journal, 2012, 2012, 1-12.

[9] MC. Chartier-Harlin, J. Kachergus, C. Roumier, V. Mouroux, X. Douay, S. Lincoln and C. Levecque, $\alpha$-synuclein locus duplication as a cause of familial Parkinson's disease. The Lancet. 364(9440), 2004, 1167-1169.

[10] A. Zimprich, S. Biskup, P. Leitner, P. Lichtner and M. Farrer, Mutations in LRRK2 cause autosomal-dominant parkinsonism with pleomorphic pathology, Neuron, 44(4), 2004, 601-607.

[11] Y. Liu, L. Fallon, HA. Lashuel, Z. Liu and PT Lansbury. The UCH-L1 gene encodes two opposing enzymatic activitiesthat affect $\alpha$ synuclein degradation and Parkinson's disease susceptibility, Cell,111(2), 2002, 209-18.

[12] C. Lautier, S. Goldwurm and A Durr. Mutations in theGIGYF2 (TNRC15) gene at the PARK11 locus in familial Parkinson disease, American Journal of Human Genetics, 82(4), 2008, 822-833.

[13] KM. Strauss, LM. Martins and H. Plun-Favreau, Loss of function mutations in the gene encoding Omi/HtrA2 in Parkinson's disease, Human Molecular Genetics, 14(15), 2005, 2099-2111.

[14] M. Kitada, S. Asakaw and N. Hattori. Mutations in theparkin gene cause autosomal recessive juvenile parkinsonism, Nature, 392(6676), 605-608

[15] EM. Valente, PM. Abou-Sleiman and V. Caputo. Hereditaryearly-onset Parkinson's disease caused by mutations in PINK1,Science, 304(5674), 1158-1160.

[16] PJ. Lockhart, S. Lincoln and M. Hulihan M, DJ-1 mutationsare a rare cause of recessively inherited early onset parkinsonismmediated by loss of protein function," Journal of Medical Genetics,41(3), 2004, 1-22.

[17] DJ. Hampshire, E. Roberts and Y, Crow, Kufor-Rakeb syndrome,pallido-pyramidal degeneration with supranuclearmupgaze paresis and dementia,maps to 1p36, Journal of Medical Genetics, 38(10), 2001, 680-682.

[18] C, Paisan-Ruiz, R. Guevara and M. Federoff, Early-onset Ldopa-responsive Parkinsonism with pyramidal signs due to ATP13A2, PLA2G6, FBXO7 and Spatacsin mutations, Movement Disorders, 25(12), 2010, 1791-1800.

[19] S. Lesage and A. Brice, Parkinson's disease: from monogenic forms to genetic susceptibility factors. Human Molecular Genetics, 18(Rl), 2009, R48-R59.

[20] I. Mata, A. Samii and S. Factor, Variation in the Alpha-Synuclein Gene, Independent of REPI, Modifies Risk for Parkinson's Disease. Neurology, 72(11), 2009, A393-A394.

[21] DL. Fortin, MD. Troyer, K. Nakamura, S. Kubo, MD. Anthony, and RH. Edwards, Lipid rafts mediate the synaptic localization of alpha-synuclein. Journal of Neuroscience, 24(30), 2004, 6715-6723.

[22] MH. Polymeropoulos, C. Lavedan, E. Leroy, SE. Ide, A. Dehejia, A. Dutra, B. Pike, H. Root, J. Rubenstein, R. Boyer, ES. Stenroos, and S. Chandrasekharappa, Mutation in the alpha-synuclein gene identified in families with Parkinson's disease, Science, 276(5321), 1997, 2045-2047.

[23] P. Ibanez, S. Lesage, S. Janin, E. Lohmann, F. Durif, A. Destée, AM. Bonnet, C. Brefel-Courbon, and S. Heath, Alpha-synuclein gene rearrangements in dominantly inherited parkinsonism: frequency, phenotype, and mechanisms, Archives of Neurology,66(1), 2009, 102-108.

[24] K. Seidel, L. Schols, S. Nuber, E. Petrasch-Parwez, K. Gierga, Z. Wszolek, D. Dickson, WP. Gai, A. Bornemann, O. Riess, A. Rami, WF. Den-Dunnen, T. Deller, U. Rüb and R. Krüger, First appraisal of brain pathology owing to A30P mutant alphasynuclein. Annals of Neurology,67(5), 2010, 684-689. 
[25] MB. Lynn, FM. Ignacio and PZ. Cyrus, The Genetics of Parkinson Disease. Journal of Geriatric and Psychiatry Neurology, 23(4), $2010,228-242$.

[26] K. Nuytemans, J. Theuns, M. Cruts and C. Broeckhoven, Genetic etiology of Parkinson disease associated with mutations in the SNCA, PARK2, PINK1, PARK7, and LRRK2 genes: A mutation update, Human Mutation ,31(7), 2010, 763-780.

[27] S. Lesage, A. Durr, M. Tazir, E. Lohmann, AL. Leutenegger, S. Janin, P. Pollak, A. Brice, LRRK2 G2019S as a cause of Parkinson's disease in North African Arabs. The New England Journal of Medicine, 354(4), 2006, 422-423.

[28] LN. Clark, Y. Wang, E. Karlins, L. Saito, H. Mejia-Santana, J. Harris, ED. Louis, LJ. Cote, H. Andrews, and S. Fahn, Frequency of LRRK2 mutations in early- and late-onset Parkinson disease, Neurology, 67(10), 2006, 1786-1791.

[29] CP. Zabetian, CM. Hutter, D. Yearout, AN. Lopez, SA. Factor, A. Griffith, BC. Leis, TD. Bird, JG. Nutt, DS.Higgins, JW. Roberts, DM. Kay, KL. Edwards, A. Samii, and H. Payami, LRRK2 G2019S in Families with Parkinson Disease Who Originated from Europe and the Middle East: Evidence of Two Distinct Founding Events Beginning Two Millennia Ago, American Journal of Human Genetics, 79(4), 2006, 752-758.

[30] LJ. Ozelius, G. Senthil, R. Saunders-Pullman, E. Ohmann, A. Deligtisch, M. Tagliati, AL. Hunt, C. Klein, B. Henick, SM. Hailpern, LRRK2 G2019S as a cause of Parkinson's disease in Ashkenazi Jews, The New England Journal of Medicine, 354(4), 2006, 424-425.

[31] H. Shimura, N. Hattori, S. Kubo, Y. Mizuno, S. Asakawa, S. Minoshima, N. Shimizu, K. Iwai, T. Chiba, K. Tanaka, Familial Parkinson disease gene product, parkin, is a ubiquitin-protein ligase, Nature Genetics, 25(3), 2000, 302-305.

[32] S. Asakawa, KI. Tsunematsu, A. Takayanagi, T. Sasaki, A.Shimizu, A. Shintani, K. Kawasaki, AJ. Mungall, S. Beck, S. Minoshima and N. Shimizu, The genomic structure and promoter region of the human parkin gene. Biochemical and Biophysical Research Communication, 286(5), 2001, 863-868

[33] CB. Lucking, A. Durr and V. Bonifati. Association between early-onset Parkinson's disease and mutations in the parkin gene. The New England Journal of Medicine, 342(21), 2000, 1560-1567.

[34] H. Takahashi, E. Ohama and S. Suzuki, Familial juvenile parkinsonism: clinical and pathologic study in a family, Neurology, 44(3 pt 1), 1994, 437-441.

[35] H. Mori, T. Kondo and M. Yokochi. Pathologic and biochemical studies of juvenile parkinsonism linked to chromosome 6q, Neurology, 51(3), 1998, 890-892.

[36] K. Hedrich, K. Marder and J. Harris, Evaluation of 50 probands with early-onset Parkinson's disease for Parkin mutations, Neurology, 58(8), 2002, 1239-1246.

[37] M. Kann, H. Jacobs and K. Mohrmann. Role of parkin mutations in 111 community-based patients with early-onset parkinsonism. Annals of Neurology,51(5), 2002, 621-625.

[38] K. Hedrich, K. Eskelson and B. Wilmot, Distribution, type, and origin of Parkin mutations: review and case studies. Movment Disorders, 19(10), 2004, 1146-1157.

[39] V. Bonifati, P. Rizzu, MJ. Baren, O. Schaap, GJ. Breedveld, E. Krieger, MC. Dekker, F. Squitieri, P. Ibanez, M. Joosse, J.W. Dongen, N. Vanacore, JC. Swieten, A. Brice, G. Meco, CM. Duijn and BA. Oostra, Mutations in the DJ-1 gene associated with autosomal recessive early-onset parkinsonism, Science, 299(5604), 2003,256-259.

[40] H. Xiong, D. Wang, L. Chen, YS. Choo, H. Ma, C. Tang, K. Xia, W. Jiang, Z. Ronai, X. Zhuang and Z. Zhang, PINK1, and DJ-1 form a ubiquitin E3 ligase complex promoting unfolded protein degradation. Journal of Clinical Investigation, 119(3), 2009, 650660 .

[41] D. Nagakubo, T. Taira and H. Kitaura, DJ-1, a novel oncogene which transforms mouse NIH3T3 cells in cooperation with ras. Biochemical and Biophysics Research Communication. 231(2), 1997, 509-513.

[42] JA. Olzmann, K. Brown, KD. Wilkinson, HD. Rees, Q. Huai, H. Ke, AI. Levey, L. Li and LS. Chi, Familial Parkinson's diseaseassociated L166P mutation disrupts DJ-1 protein folding and function, Journal of Biological Chemistry, 279(9), 2004, 8506-8515.

[43] PC. Anderson and V. Daggett, Molecular basis for the structural instability of human DJ-1 induced by the L166P mutation associated with Parkinson's disease. Biochemistry, 47(36), 2008, 9380-9393.

[44] EM. Valente, AR. Bentivoglio and PH Dixon, Localization of a novel locus for autosomal recessive early-onset parkinsonism, PARK6, on human chromosome 1p35-p36, American Journal of Human Genetics, 68(4), 2001, 895-900.

[45] S. Kawajiri, S. Saiki, S. Sato, and N. Hattori, Genetic mutations and functions of PINK1, Trends in Pharmacological Sciences, 32(10), 2011, 573-580.

[46] V. Bonifati, CF. Rohe and GJ. Breedveld. Early-onset parkinsonism associated with PINK1 mutations: frequency, genotypes, and phenotypes, Neurology, 65(1), 2005, 87-95.

[47] Y. Hatano, Y. Li, K. Sato, S. Asakawa, Y. Yamamura, H. Tomiyama, H. Yoshino ,M. Asahina, S. Kobayashi, S. Hassin-Baer, CS. Lu, AR. Ng, RL. Rosales, N. Shimizu, T. Toda, Y. Mizuno, N. Hattori. Novel PINK1 mutations in early-onset Parkinsonism. Annals of Neurology, 56(3), 2004, 424-7.

[48] PM. Abou-Sleiman, MM. Muqit, NQ. McDonald, YX, Yang, S. Gandhi, DG. Healy, K. Harvey, RJ. Harvey, E. Deas, K. Bhatia, N. Quinn, A. Lees, DS. Latchman, and NW. Wood, A heterozygous effect for PINK1 mutations in Parkinson's disease? Annals of Neurology, 60(4), 2006, 414-419.

[49] EK.Tan and LM. Skipper LM. Pathogenic mutations in Parkinson disease. Human Mutation, 28(7), 2007,641-653.

[50] A. Ramirez, A. Heimbach and J. Grundemann, Hereditary parkinsonism with dementia is caused by mutations in ATP13A2, encoding a lysosomal type 5 P-type ATPase, Nature Genetics, 38(10), 2006, 1184-1191.

[51] J. Tan, T. Zhang, L. Jiang, J. Chi, D. Hu, Q. Pan, D. Wang and Z Zhang, "Regulation of intracellular manganese homeostasis by Kufor-Rakeb syndrome-associated ATP13A2 protein, Journal of Biological Chemistry, 286(34), 2011, 29654-29662.

[52] R. Myhre, S. Steinkjer and A. Stormyr, Significance of the parkin and PINK1 gene in Jordanian families with incidences of you ngonset and juvenile parkinsonism. BMC Neurology, 8(1), 2008, 47.

[53] FA. Di, HF. Chien and M. Socal, ATP13A2 missense mutations in juvenile parkinsonism and young onset Parkinson disease, Neurology, 68(19), 2007,1557-1562.

[54] N. Pankratz, WC. Nichols, SK. Uniacke, C.Halter, A. Rudolph, C. Shults, PM. Conneally and T. Foroud, Significant linkage of Parkinson disease to chromosome 2q36-37. American Journal of Human Genetic, 72(4), 2003a,1053-1057.

[55] B. Giovannone, E. Lee, L. Laviola, F. Giorgino, KA. Cleveland and RJ. Smith, Two novel proteins that are linked to insulin-like growth factor (IGF-I) receptors by the Grb10 adapter and modulate IGF-I signaling, Journal of Biological Chemistry, 278(34), 2003, 31564-31573.

[56] J. Bras, J. Simon-Sanchez, M. Federoff, A. Morgadinho, C. Januario, M. Ribeiro, L. Cunha, C. Oliveira and AB. Singleton, Lack of replication of association between GIGYF2 variants and Parkinson disease, Human Molecular Genetics, 18(2), 2009, 341-346.

[57] OA. Ross, AI. Soto, C. Vilarino-Guell, MG. Heckman, NN. Diehl, MM. Hulihan, JO. Aasly, S. Sando, JM. Gibson and T. Lynch, Genetic variation of Omi/HtrA2 and Parkinson's disease. Parkinsonism Related Disorder, 14, 2008, 539-543. 
[58] S. Shojaee, F. Sina, SS. Banihosseini, MH. Kazemi, R. Kalhor, GA. Shahidi, H. Fakhrai-Rad, M. Ronaghi and E. Elahi. Genomewide linkage analysis of a Parkinsonian-pyramidal syndrome pedigree by $500 \mathrm{~K}$ SNP arrays. American Journal of Human Genetics, 82(6), 2008, 1375-1384.

[59] MS. Ho, C. Ou, YR. Chan, CT. Chien and H. Pi, The utility F-box for protein destruction. Cellular and Molecular Life Sciences, 65(13), 2008, 1977-2000.

[60] C. Paisan-Ruiz, KP. Bhatia, A. Li, D. Hernandez, M. Davis, NW. Wood, J. Hardy, H. Houlden , A. Singleton and Schneider, Characterization of PLA2G6 as a locus for dystonia-parkinsonism. Annals of Neurology, 65(1), 2009, 19-23.

[61] KS. Hruska, ME. LaMarca, CR. Scott and E. Sidransky, Gaucher disease: mutation and polymorphism spectrum in the glucocerebrosidase gene (GBA), Human Mutation.29(5), 2008, 567-583.

[62] N. Pankratz, WC. Nichols, SK. Uniacke, C. Halter, J. Murrell, A. Rudolph, CW. Shults, PM. Conneally and T. Foroud, Genomewide linkage analysis and evidence of gene-by-gene interactions in a sample of 362 multiplex Parkinson disease families. Human Molecular Genetics, 12(20), 2003b,2599-2608.

[63] Gasser T., Muller-Myhsok B., Wszolek Z.K., Oehlmann R.,Calne D.B., Bonifati V., Bereznai B., Fabrizio E. Vieregge P., Horstmann R.D.A susceptibility locus for Parkinson's disease maps to chromosome 2p13. Nature Genetics, 18(3), 1998, 262-265.

[64] AA. Hicks, H. Petursson, T. Jonsson, H. Stefansson, HS. Johannsdottir, J. Sainz, ML. Frigge, A. Kong, JR. Gulcher and K. Stefansson, A susceptibility gene for late-onset idiopathic Parkinson's disease. Annals of Neurology,52(5), 2002,549-555.

[65] SC. Zhang, M. Wernig, ID. Duncan, O. Brustle and JA. Thomson, In vitro differentiation of transplantable neural precursors from human embryonic stem cells, Nature Biotechnology, 19, 2001, 1129-1133.

[66] D. Lau, U. Ogbogu, B. Taylor, T. Stafinski, D. Menon and T. Caulfield. Stem cell clinics online: the direct-to consumer portrayal of stem cell medicine. Cell Stem Cell, 3(6), 2008, 591-594.

[67] PH. Schwartz, DJ. Brick, AE. Stover, JF. Loring and FJ. Muller, Differentiation of neural lineage cells from human pluripotent stem cells, Methods. 45, 2008, 142-158.

[68] K. Tanaka, T. Suzuki, N. Hattori and Y. Mizuno, Ubiquitin, proteasome and parkin. Biochimica et Biophysica Acta. 1695(1-3), 2004, 235-247.

[69] A. Hermann, M. Maisel and S. Liebau. Mesodermal celltypes induce neurogenesis from adult human hippocampal progenitor cells. Journal of Neurochemistry, 98(2), 2006, 629-640.

[70] I. Hamaguchi, Lentivirus vector gene expression during ES cell-derived hematopoietic development in vitro. Journal of Virology. 74(22), 2000, 10778-10784.

[71] BY. Hu, Neural differentiation of human induced pluripotent stem cells follows developmentalprinciples but with variable potency. Proceedings of National Academy of Sciences United States of America,. 107(9), 2010, 4335-4340.

[72] DJ. Prockop, Marrow stromal cells as stem cells for non hematopoietic tissues, Science, 276(5309), 1997, 71-74.

[73] Y. Kuroda, M. Kitada and S. Wakao, Unique multipotentcells in adult human mesenchymal cell populations. Proceedings of the National Academy of Sciences of the United States of America, 107(19), 2010, 8639-8643.

[74] M. Dezawa, Insights into autotransplantation: the unexpecteddiscovery of specific induction systems in bonemarrow stromal cells, Cellular and Molecular Life Sciences, 63(23), 2006,2764-2772.

[75] M. Kitada and M. Dezawa, Parkinson's Disease and Mesenchymal Stem Cells:Potential for Cell-Based Therapy, Parkinson's Disease, 2012, 2012, 1-9.

[76] G. Bouchez, L. Sensebe and P. Vourch, Partial recovery of dopaminergic pathway after graft of adult mesenchymal stem cells in a rat model of Parkinson's disease, Neurochemistry International, 52(7), 2008, 1332-1342.

[77] M. Politis and O. Lindvall, Clinical application of stem cell therapy in Parkinson's disease, BMC Medicine, 10, $2012,1$.

[78] MK. McCoy, TN.Martinez and KA. Ruhn, Autologous transplants of Adipose-Derived Adult Stromal (ADAS) cells afford dopaminergic neuroprotection in a model of Parkinson's Disease. Experimental Neurology, 210, 2008, 14-29.

[79] YS. Fu, YC. Cheng, MY. Lin, H. Cheng, PM. Chu, SC. Chou, YH. Shih, MH. Ko and MS. Sung, Conversion of human umbilical cord mesenchymal stem cells in Wharton's jelly to dopaminergic neurons in vitro: potential therapeutic application for Parkinsonism. Stem Cells, 24(1), 2006, 115-24.

[80] HJ. Park, PH. Lee, OY. Bang, G. Lee and YH. Ahn, Mesenchymal stem cells therapy exerts neuroprotection in a progressive animal model of Parkinson's disease, Journal of Neurochemistry, 107(1), 2008, 141-51.

[81] JA. Thomson, Embryonic stem cell lines derived from human blastocysts. Science. 282(5391), 1998, 1145-1147.

[82] H. Fukuda, J. Takahashi and K. Watanabe. Fluorescenceactivated cell sorting-based purification of embryonic stem cell-derived neural precursors averts tumor formation after transplantation. Stem Cells. 24(3), 2006, 763-771.

[83] H. Li, JC. Radford, MJ. Ragusa, KL. Shea, and SR. McKercher, Transcription factor MEF2C influences neural stem/progenitor cell differentiationand maturation in vivo. Proceedings of the National Academy of Sciences of the United States of America, 105(27), 2008, 9397-9402.

[84] X. Zeng, J. Cai, J. Chen, Y. Luo, ZB. You, E. Fotter, Y. Wang, B. Harvey, T. Miura, C. Backman, GJ. Chen, MS. Rao, and WJ. Freed, Dopaminergic differentiation of human embryonic stem cells, Stem Cells, 22(6), 2004, 925-40.

[85] JA. Rodriguez-Gomez, JQ. Lu, I. Velasco, S. Rivera, SS. Zoghbi and JS. Liow, Persistent dopamine functions of neurons derived from embryonic stem cells in a rodent model of Parkinson disease, Stem Cells, 25(4), 2007, 918-28.

[86] C. Eun-Gyung, DZ. Jeffrey, RM. Scott, T. Maria, T. Shichun, M. Eliezer, FC. Shing, N. Nobuki, T. Alexey and AL. Stuart, MEF2C Enhances Dopaminergic Neuron Differentiationof Human Embryonic Stem Cells in a Parkinsonian Rat Model. PLoS ONE. 6, 2011, 24027.

[87] I. Datta, S. Mishra, L. Mohanty, S. Pulikkot and PG. Joshi, Neuronal plasticity of human Wharton's jelly mesenchymalstromal cells to the dopaminergic cell type compared withhuman bone marrow mesenchymal stromal cells. Cytotherapy, 13(8), 2011, 918-932.

[88] T. Deierborg, D. Soulet, L. Roybon, V. Hall and P. Brundin. Emerging restorative treatments for Parkinson's disease. Progress in Neurobiology, 85(4), 2008, 407-432.

[89] C. Laker, Host cis-mediated extinction of a retrovirus permissive for expression in embryonal stem cells during differentiation. Journal of Virology, 72(1), 1998, 339-348.

[90] M. Deleidi, O. Cooper, G. Hargus, A. Levy and O. Isacson O, Oct4-Induced Reprogramming Is Required for Adult Brain Neural Stem Cell Differentiation into Midbrain Dopaminergic Neurons, PLoS On, 6(5), 2011, 19926.

[91] YX. Ding, LC. Wei, YH. Liu, L. Duan and XY. Jiao. Midbrain Neural Stem Cells Show Unique Cell Survival, Neuronal Commitment and Neurotrophic Properties with Therapeutic Potential for Parkinson's Disease. Journal of Alzheimers Disease, 10, 2012, 001.

[92] K. Takahashi, K. Tanabe and M. Ohnuki, Induction ofpluripotent stemcells from adult human fibroblasts by defined factors, Cell, 131(5), 2007, 861-872. 
[93] R. Yong-Hee, k. Ji-Yun, C. Mi-Yoon, Y. Sang-Hoon, K. Dohoon, K. Chun-Hyung, S. Jae-Won, J. A-Young,K. Byung-Woo and L. Hyunsu, Protein-based human iPS cells efficiently generate functional dopamine neurons and can treat a rat model of Parkinson disease, The Journal of Clinical Investigation,121(6), 2011, 2326-2335.

[94] K. Okita, M. Nakagawa, H. Hyenjong, TY. Ichisaka and S. Amanaka. Generation of mouse induced pluripotentstem cells without viral vectors. Science, 322(5903), 2008, 949-953.

[95] O. Cooper, G. Hargus, M. Deleidi, A. Blak ,T. Osborn, E. Marlow, K. Lee, A. Levy, E. Perez-Torres, A. Yow and O.Isacson. Differentiation of human ES and Parkinson's disease iPS cells into ventral midbrain dopaminergic neurons requires a high activity form of SHH, FGF8a and specific regionalization by retinoic acid, Molecular Cell Neuroscience, 45(3), 2010, 258-266. 